\title{
Antifungal activity of dual combination of hydroxychavicol with commercialized agents against oral Candida species
}

Wan Harun Himratul-Aznita ${ }^{1 *}$, Che Omran Nor-Zulaila ${ }^{2}$ and Khairuddin Nurul-Fatihah ${ }^{2}$

\begin{abstract}
Candida spp. is the most prevalent species causing systemic fungal infections. The effect of antifungal agents were screened in vitro and their synergism effect were determined between hydroxychavicol $(\mathrm{HC})$ in association with commercialized antifungal drugs-amphotericin B (AMB), and 5-fluorocytosine (5-FC) alone and in combination against five different oral Candida spp. in their planktonic states at different ratio $(1: 1 \mathrm{v} / \mathrm{v} ; 1: 2 \mathrm{v} / \mathrm{V}$ and $2: 1 \mathrm{v} / \mathrm{v})$. In vitro susceptibilities of Candida spp. to HC and commercialized antifungal agents were investigated by broth microdilution method as described by Clinical and Laboratory Standards Institute M38-A2. The intensity of the interactions was evaluated by visual reading and spectrophotometric method in checkerboard microdiluton assay, and the nature of the interactions was assessed by fractional inhibitory concentration index. The minimum inhibitory concentration $\left(\mathrm{MIC}_{50}\right)$ of $\mathrm{HC}, \mathrm{AMB}$ and 5-FC alone against five different planktonic oral Candida spp. ranged from 240 to 120, 8 to 15 , and 2 to $8 \mu \mathrm{g} / \mathrm{mL}$ respectively. Positive synergistic effect existed between $\mathrm{HC}$ and $\mathrm{AMB}$ at 1:1 ratio in all Candida spp. However, there was no synergy effect observed in the majority of Candida spp. for the combination of HC with 5-FC. The data of combination between $\mathrm{HC}$ with AMB may be useful in the treatment of systemic infections caused by oral Candida spp. instead of the combination of HC with 5-FC.
\end{abstract}

Keywords: Candida, Hydroxychavicol, Amphotericin B, Synergistic

\section{Background}

Invasive fungal infections, such as candidiasis, represent a public health problem of major importance. Since its discovery in 1839 by Langenbeck, the genus Candida has been shown to be the causative agent of many infections and represent a component of the normal flora in the oral cavity (Fridkin and Jarvis 1996). According to previous study, candidal adherence to mucosal surfaces is considered as a critical initial step in the pathogenesis of oral candidiasis (Bokor-Bratic 2008).

The high global incidence and prevalence of oral candidiasis may be attributed to an increasing usage of broad-spectrum antibiotics, cytotoxic, corticosteroids, and to a growing number of immuno-suppressed

\footnotetext{
*Correspondence: aznita@um.edu.my

1 Department of Oral Biology and Craniofacial Sciences, Faculty of Dentistry, University of Malaya, 50603 Kuala Lumpur, Malaysia Full list of author information is available at the end of the article
}

individuals as well as those with common endocrine disorders (such as diabetes mellitus) or severe nutritional deficiencies (Johnson et al. 2004). The increasing incidence of fungal infections without a satisfactory response to the current antifungal therapy and the slow development of new agents with novel mechanisms of action have produced significant interest on associations between antifungal agents (Hemaiswaryaa et al. 2008).

$\mathrm{HC}$ is the major phenolic component, isolated from the aqueous extract of $P$. betle L., leafand has been reported to exhibit antibacterial activities against oral cavity pathogens (Sharma et al. 2009) by inhibit the growth and disrupt the permeability barrier of microbial membrane structures. However, the report on its antifungal activity is lacking.

Polyenes such as AMB (isolated from Streptomycin spp.) bind to ergosterol and disrupt the major lipid component of the fungal cell membrane. From 1950s until the discovery of the azoles, polyenes antifungal agents 
represented the standard of therapy for systemic fungal infections (Sugar 1986). While, flucytosine (5-FC) is a synthetic antimycotic compound and possess no intrinsic antifungal capacity. It will be converted into 5-fluorouracil (5-FU) once been taken up by susceptible fungal cells, and is further converted to metabolites that inhibit fungal RNA and DNA synthesis (Vermes et al. 2000).

Some of the most effective antifungal drugs are too toxic for continuous use or can only be administered intravenously (Ghannoum and Rice 1999). The ideal antifungal drug would be non-toxic, fungicidal, and amenable to self-administration. Combination therapy is one approach that can be used to improve the efficacy of antimicrobial therapy. Thus, the present study was carried out with the aim of investigating the combination of $\mathrm{HC}$ with AMB and 5-FC against planktonic of oral associated Candida spp.

\section{Methods}

\section{Candida strains and growth condition}

Five strains of Candida spp. used in this study were purchased from The American Type Culture Collection (ATCC), USA. The species were C. albicans ATCC 14053, C. tropicalis ATCC 13803, C. parapsilosis ATCC 22019, C. lusitaniae ATCC 64125 and C. dubliniensis ATCC MYA-2975. Upon revival, each respective Candida strain was stored in $20 \% \mathrm{v} / \mathrm{v}$ glycerol and kept in $-70{ }^{\circ} \mathrm{C}$. All strains were also subcultured monthly on Yeast Peptone Dextrose (YPD) agar media (BD Difco, USA) and maintained weekly at $4{ }^{\circ} \mathrm{C}$ throughout the experimental period. Purity of the phenotypes was further confirmed by API Yeast Identification System (API 20C Aux, BioMerieux, France). Throughout the experiment period, the cultures were maintained on YPD agar up to a maximum of 2 weeks at $4{ }^{\circ} \mathrm{C}$. Regular sub-culturing was carried out every 2 weeks to maintain viability of the cells.

\section{Preparation of the standard candida cell suspension, bioactive compound and antifungal agents}

Each Candida strain was respectively cultured on Yeast Peptone Dextrose (YPD) agar media (BD Difco, USA) at $37{ }^{\circ} \mathrm{C}$ for $24 \mathrm{~h}$ according to Harun et al. (2014). The turbidity of the suspension was adjusted to an optical density $\left(\mathrm{OD}_{550 \mathrm{~nm}}\right)$ of 0.144 which is equivalent to $1 \times 10^{6}$ cells $/ \mathrm{mL}$. HC was isolated in the pure form from the chloroform extraction of the aqueous leaf extract of $P$. betle L., (Piperaceae) as described previously (Sharma et al. 2009). AMB and 5-FC were obtained as standard liquid and powders purchased from Sigma Chemical Co. (St. Louis, MO).

A stock solution of $1000 \mu \mathrm{g} / \mathrm{mL} \mathrm{HC}$ and $250 \mu \mathrm{g} / \mathrm{mL}$ AMB was prepared in $5 \% \mathrm{DMSO}$ and stored at $4{ }^{\circ} \mathrm{C}$ until used. A $5 \%$ DMSO did not influence the growth and viability of fungi in a pilot study carried out prior to the experiment in our laboratory. A stock solution of $250 \mu \mathrm{g} /$ $\mathrm{mL}$ of 5 -FC was prepared in sterile distilled water. AMB was also used in the study as the positive control.

\section{Determination of MIC}

The minimum inhibitory concentrations (MIC) of active compound and antifungal agentsalone against Candida spp. was determined according to the microdilution method of the CLSI M38-A2using 96-well microtiter plates with some modifications (Clinical and Laboratory Standards Institute 2008). This modification involved the usage of YPD instead of Sabaroud Dextrose Agar (SDA) or Potato Dextrose Agar (PDA) as describe in Harun et al. (2014). Concentration of $1 / 2$ MIC was used throughout the study as the research was focused in controlling the population of Candida spp. in the oral cavity rather than giving a complete killing effect, considering Candida spp. as oral commensals. A broth microdilution method was recommended by CLSI as a general standard methodology for testing active compound or commercialized antifungal agent. Thus, this method was employed to analysed the MIC of HC, AMB and 5-FC.

Each well contained the $10 \mu \mathrm{L}$ of Candida spp. at a final concentration of $1.0 \times 10^{6}$ cells $/ \mathrm{mL}, 100 \mu \mathrm{L}$ of YPD broth, and $100 \mu \mathrm{L}$ of $\mathrm{HC}$, AMB and 5-FC. YPD broth without test agents was included as an agent-free control, and YPD broth was used as medium blank. All plates were incubated in an aerobic incubator at $37^{\circ} \mathrm{C}$ for $24 \mathrm{~h}$, after which the growth was determined spectrophotometrically at $550 \mathrm{~nm}$ by means of a microplate reader (PowerWave 200, Bio-Tek Instruments, and Winooski, VT, USA). The data were reported as the median of at least 3 independent tests.

\section{Assessment of $\mathrm{HC}$ and commercialized antifungal agents against Candida spp}

Synergistic effects of $\mathrm{HC}$ to $\mathrm{AMB}$ and $\mathrm{HC}$ to 5 - $\mathrm{FC}$ at a ratio of $1: 1 \mathrm{v} / \mathrm{v}, 1: 2 \mathrm{v} / \mathrm{v}$ and $2: 1 \mathrm{v} / \mathrm{v}$ against five planktonic of oral Candida spp. were quantitatively determined using the checkerboard microdilution method as described in previous study (Cuenca-Estrella 2004).

Briefly, $1000 \mu \mathrm{L} \mathrm{HC}, 250 \mu \mathrm{L}$ AMB and $250 \mu \mathrm{L} 5-\mathrm{FC}$ were used as initial concentration in this study. A 1:1 ratio of either $\mathrm{HC}$ and $\mathrm{AMB}$ or $\mathrm{HC}$ and 5-FC was prepared. Firstly, $50 \mu \mathrm{L}$ of $\mathrm{HC}$ was added into well 1 of 96 microtitre plate. Followed by an addition of $50 \mu \mathrm{L}$ of either AMB. It was then serially diluted up to well 12 . Inoculum of $1 \times 10^{6}$ cells $/ \mathrm{mL}$ was added to each well and the plates were incubated at $37{ }^{\circ} \mathrm{C}$ for $24 \mathrm{~h}$ and read spectrophotometrically at $550 \mathrm{~nm}$ using a microplate reader (PowerWave 200, Bio-Tek Instruments, and Winooski, VT, USA). Negative control was prepared without the 
addition of $\mathrm{HC}$ and AMB. Similar procedure was carried out for 1:1 ratio of $\mathrm{HC}$ and 5-FC. The analysis was also performed with 1:2 and 2:1 ratio of $\mathrm{HC}: \mathrm{AMB}$ and HC:5-FC.

The MIC combination was determined as the concentrations of antifungal drugs that resulted a $50 \%$ reduction in absorbance compared to that of control. The MIC values of combined $\mathrm{HC}$ with $\mathrm{AMB}$ and 5-FC used to determine the fractional inhibitory concentration (FIC). The FIC index ( $\sum$ FIC, the sum of individual FICs) was calculated using the formula: $\sum \mathrm{FIC}=\mathrm{MIC}\left(\mathrm{A}_{\text {comb }}\right) / \mathrm{MIC}$ $\left(\mathrm{A}_{\text {alone }}\right)+\mathrm{MIC}\left(\mathrm{B}_{\text {comb }}\right) / \mathrm{MI}$ C $\left(\mathrm{B}_{\text {alone }}\right)$.

Two drugs or bioactive compounds are defined as having synergistic effect, if the FIC indexes are $\leq 0.5$, whereas they are said to have indifference when the FIC 0.5 but $\leq 4$, and antagonistic when FIC index was $>4$ (CuencaEstrella 2004).

\section{Results}

$\mathrm{MIC}_{50}$ of HC, AMB and 5-FC against oral Candida spp

The inhibitory activities of HC, AMB and 5-FC against a series of oral-associated Candida spp. were investigated (Table 1). Planktonic growth of Candida spp. was susceptible to different antifungal drugs at varying concentrations. The $\mathrm{MIC}_{50}$ range of $\mathrm{HC}$ against Candida spp. after $24-\mathrm{h}$ incubation was subsequently $240-120 \mu \mathrm{g} / \mathrm{mL}$. It has been found that, C. parapsilosis and C. dubliniensis were the most susceptible to $\mathrm{HC}$.

The MICs of individual AMB and 5-FC against the test strains of Candida species were lower than those of $\mathrm{HC}$, ranging from 8 to 15 and 2 to $8 \mu \mathrm{g} / \mathrm{mL}$, respectively.

$\mathrm{AMB}$ alone was active against $C$. tropicalis and $C . d u b$ liniensis at MIC of $4 \mu \mathrm{g} / \mathrm{mL}$, C. albicans and C. parapsilosis at MIC, of $8 \mu \mathrm{g} / \mathrm{mL}$ and C. lusitaniae at MIC of $15 \mu \mathrm{g} /$ $\mathrm{mL}$.

5-FC alone was active against $C$. albicans, $C$. parapsilosis and $C$. dubliniensis at $2 \mu \mathrm{g} / \mathrm{mL}$ of MIC concentration, while $C$. lusitaniae and C. tropicalis was slightly less susceptible to 5 -FC at 4 and $8 \mu \mathrm{g} / \mathrm{mL}$ of MIC.

\section{The effect of HC/AMB on the planktonic growth of Candida spp}

Checkerboard analysis, has determined that the combination of $\mathrm{HC}$ and AMB having MIC of synergistic effect against five planktonic Candida spp. with $\sum$ FIC index $\geq 0.5$. The FIC index for $\mathrm{HC}$ in combination with $\mathrm{AMB}$ was calculated as shown in Tables 1, 2 and 3. The combination $\mathrm{HC} / \mathrm{AMB}$ at 1:1 ratio yielded synergism interaction against all five Candida species, while at 1:2 and 2:1 ratio of combination, C. tropicalis and C. lusitaniae showed indifferent effect.

At 1:1, 1:2 and 2:1 ratio of HC/AMB against C. albicans, the MIC of HC were decreased up to 120- to 60-fold, while MIC of AMB were decreased about 8- to 16-fold. As shown in Table 1, strong synergistic effect has been demonstrated at 1:1 and 2:1 ratio with the FIC index of 0.07 .

Against C. parapsilosis, strong synergistic effect has been observed at 2:1 ratio of $\mathrm{HC} / \mathrm{AMB}$ with the FIC index of 0.08 , where the MIC combination was markedly decreased (60- to 4-fold respectively). However, at 1:1 and 1:2 ratios, the synergism effect was also observed with the FIC index of 0.16 , which is slightly higher than those observed in 2:1 ratio.

As illustrated in Table 1, a synergistic effect was observed against $C$. tropicalis (FICI, 0.27) at 1:1 ratio, however showed indifferent effect in 2:1 (FICI, 0.53) and 1:2(FICI, 1.06) ratios. MIC of $\mathrm{HC} / \mathrm{AMB}$ in 1:1 ratio has shown decreased of about 60 -fold and 8 -fold respectively (Table 1).

Against C. dubliniensis, the MIC of $\mathrm{HC}$ in association with AMB at 1:1 ratio decreased about 60:8-fold (FICI, 0.26 ) where synergism was observed. Differently, a synergism was also found at 1:2 and 2:1 ratio with FIC index

Table 1 Susceptibilities of standard Candida spp. to HC alone and in combination with AMB and 5-FC (1:1) ratio

\begin{tabular}{|c|c|c|c|c|c|c|c|c|c|c|c|}
\hline \multirow[t]{2}{*}{ Species } & \multicolumn{3}{|c|}{ MIC $(\mu \mathrm{g} / \mathrm{mL})$ alone $\mathrm{a}^{\mathrm{a}}$} & \multicolumn{8}{|c|}{ MIC $(\mu \mathrm{g} / \mathrm{mL})$ combination $(1: 1)$ ratio $^{a}$} \\
\hline & $\mathrm{HC}$ & Amp B & $5-\mathrm{FC}$ & $\mathrm{HC}^{\mathbf{b}}$ & Amp B & $\mathrm{HC}+\mathrm{Amp} \mathrm{B} \mathrm{FICl}$ & INT & $\mathrm{HC}^{\mathrm{c}}$ & $5-\mathrm{FC}$ & $\mathrm{HC}+5-\mathrm{FC} \mathrm{FICl}$ & INT \\
\hline C. albicans & 240 & 8 & 2 & 2 & 0.5 & 0.07 & SYN & 8 & 2 & 1.03 & IND \\
\hline C. parapsilosis & 120 & 8 & 2 & 4 & 1 & 0.16 & SYN & 15 & 4 & 2.04 & IND \\
\hline C. tropicalis & 240 & 4 & 8 & 4 & 1 & 0.27 & SYN & 30 & 8 & 1.13 & IND \\
\hline C. dubliniensis & 120 & 4 & 2 & 2 & 0.5 & 0.26 & SYN & 15 & 4 & 2.13 & IND \\
\hline C. Iusitaniae & 240 & 15 & 4 & 8 & 2 & 0.17 & SYN & 8 & 2 & 0.53 & IND \\
\hline
\end{tabular}

$\mathrm{MIC}$ and $\mathrm{FICl}$ values are shown as a mean of three independent experiments

ANT antagonism, IND indifference, SYN synergy, INT interpretation

a The MIC end point is based on the lowest drug concentration producing a decrease of $50 \%$ inhibition of fungal growth compared to untreated

b MIC of $\mathrm{HC}$ when in combination with $\mathrm{AMB}$

c MIC of HC when in combination with 5-FC 
Table 2 Susceptibilities of standard Candida spp. to HC alone and in combination with AMB and 5-FC (1:2) ratio

\begin{tabular}{|c|c|c|c|c|c|c|c|c|c|c|c|}
\hline \multirow[t]{2}{*}{ Species } & \multicolumn{3}{|c|}{ MIC $(\mu \mathrm{g} / \mathrm{mL})$ alone $^{\mathrm{a}}$} & \multicolumn{8}{|c|}{ MIC $(\mu \mathrm{g} / \mathrm{mL})$ combination $(1: 2)$ ratio $^{a}$} \\
\hline & $\mathrm{HC}$ & Amp B & $5-\mathrm{FC}$ & $\mathrm{HC}^{\mathbf{b}}$ & Amp B & $\mathrm{HC}+\mathrm{Amp} B \mathrm{FICl}$ & INT & $\mathrm{HC}^{\mathrm{c}}$ & $5-\mathrm{FC}$ & $\mathrm{HC}+5-\mathrm{FC} \mathrm{FICl}$ & INT \\
\hline C. albicans & 240 & 8 & 2 & 4 & 1 & 0.14 & SYN & 15 & 4 & 2.06 & IND \\
\hline C. parapsilosis & 120 & 8 & 2 & 4 & 1 & 0.16 & SYN & 8 & 2 & 1.06 & IND \\
\hline C. tropicalis & 240 & 4 & 8 & 15 & 4 & 1.06 & IND & 30 & 8 & 1.13 & IND \\
\hline C. dubliniensis & 120 & 4 & 2 & 4 & 1 & 0.29 & SYN & 8 & 2 & 1.06 & IND \\
\hline C. Iusitaniae & 240 & 15 & 4 & 30 & 8 & 0.67 & IND & 15 & 4 & 1.06 & IND \\
\hline
\end{tabular}

$\mathrm{MIC}$ and $\mathrm{FICl}$ values are shown as a mean of three independent experiments

ANT antagonism, IND indifference, SYN synergy, INT interpretation

a The MIC end point is based on the lowest drug concentration producing a decrease of $50 \%$ inhibition of fungal growth compared to untreated

b MIC of $\mathrm{HC}$ when in combination with $\mathrm{AMB}$

c MIC of $\mathrm{HC}$ when in combination with 5-FC

Table 3 Susceptibilities of standard Candida spp. to HC alone and in combination with AMB and 5-FC (2:1) ratio

\begin{tabular}{|c|c|c|c|c|c|c|c|c|c|c|c|}
\hline \multirow[t]{2}{*}{ Species } & \multicolumn{3}{|c|}{ MIC $(\mu \mathrm{g} / \mathrm{mL})$ alone ${ }^{\mathrm{a}}$} & \multicolumn{8}{|c|}{$\operatorname{MIC}(\mu \mathrm{g} / \mathrm{mL})$ combination (2:1) ratio ${ }^{\mathrm{a}}$} \\
\hline & $\mathrm{HC}$ & Amp B & $5-\mathrm{FC}$ & $\mathrm{HC}^{\mathbf{b}}$ & Amp B & $\mathrm{HC}+\mathrm{Amp} B \mathrm{FICl}$ & INT & $\mathrm{HC}^{\mathrm{c}}$ & $5-\mathrm{FC}$ & $\mathrm{HC}+5-\mathrm{FC} \mathrm{FICl}$ & INT \\
\hline C. albicans & 240 & 8 & 2 & 2 & 0.5 & 0.07 & SYN & 30 & 8 & $>4$ & ANT \\
\hline C.parapsilosis & 120 & 8 & 2 & 2 & 0.5 & 0.08 & SYN & 15 & 4 & 2.25 & IND \\
\hline C. tropicalis & 240 & 4 & 8 & 8 & 2 & 0.53 & IND & 30 & 8 & 1.12 & IND \\
\hline C. dubliniensis & 120 & 4 & 2 & 4 & 1 & 0.28 & SYN & 30 & 8 & $>4$ & ANT \\
\hline C. Iusitaniae & 240 & 15 & 4 & 8 & 2 & 0.17 & SYN & 15 & 4 & 1.06 & IND \\
\hline
\end{tabular}

$\mathrm{MIC}$ and $\mathrm{FICl}$ values are shown as a mean of three independent experiments

ANT antagonism, IND indifference, SYN synergy, INT interpretation

a The MIC end point is based on the lowest drug concentration producing a decrease of $50 \%$ inhibition of fungal growth compared to untreated

b MIC of $\mathrm{HC}$ when in combination with $\mathrm{AMB}$

c MIC of $\mathrm{HC}$ when in combination with 5-FC

of 0.29 and 0.28 where the MIC were decreased about 30:4-fold.

Against C. lusitaniae, it was found that a combination of $\mathrm{HC}$ and $\mathrm{AMB}$ exhibited strong synergism in 1:1 and 2:1 ratios with the FICI index of 0.17. The MIC of $\mathrm{HC}$ and AMB giving a synergistic effect was found to be 30 and 8 - fold lower than the MIC alone respectively. In comparison, the lowest activity was displayed at 1:2 with the FIC index of 0.67.

The effect of HC/5-FC on the planktonic growth of Candida spp

The MIC of the combination of $\mathrm{HC} / 5-\mathrm{FC}$ as well as the MICs of the single drug tested at the ratio of 1:1, 1:2 and 2:1, are given in Tables 1, 2 and 3. The FICI values ranged from 0.53 to 2.13 for the combination at $1: 1$ ratio, from 1.06 to 2.06 for $1: 2$ ratio and from 1.06 to $>4$ for $2: 1$ ratio.

As shown in Table 1, only C. lusitaniae of the combination of $\mathrm{HC} / 5$-FC in 1:1 ratio after 24-h incubation had partial synergistic properties with FIC index of 0.53 . Indifferent interactions were observed in C. albicans, $C$. parapsilosis, C. tropicalis and C. dubliniensis with FIC index of 2.25 and 1.06 respectively.
All of Candida spp. showed indifferent reaction at 1:2. However, antagonism has been observed for $C$. albicans and $C$. dubliniensis with FIC index $>4$ at 2:1 ratio. The MIC of 5-FC against C. albicans and C. dubliniensis increased up to fourfold. Our data showed 5-FC alone demonstrated greater effectiveness than in combination with $\mathrm{HC}$ against all Candida spp.

\section{Discussion}

Treating Candida infections with monotherapy is becoming more difficult, a major problem being the emerging drug resistance during treatment with various conventional antifungal agents. The mechanisms of antifungal resistance are categorized as primary or secondary and are related to intrinsic or acquired characteristics of the fungal pathogen, including interference with the antifungal mechanism of the respective drug or the decrease in target drug levels (Akins and Sobel 2009). Hence, there is a need to search or develop new formulation by using combinations as alternate and effective antifungal agents that have few or no side effects. The selection of the ATCC reference strains C. albicans, C. parapsilosis, 
C. tropicalis, C. dubliniensis, and C. lusitaniae was based on various reports of the prevalence of Candida species in the oral cavity (Harun et al. 2014). Although the reference strains were isolated originally from blood, similar strains have also been reported to be present in the oral cavity (Harun et al. 2014).

In recent years, the use of natural compound in combination with conventional antifungal agents to achieve drug synergy has attracted much attention. Natural compound with high effectiveness and fewer side effects are desirable as substitutes for chemical treatments which have various adverse effects. Piper betle L., (Piperaceae) has been extensively used in traditional herbal remedies in India, China, Taiwan, Thailand and many other countries. It is reported to possess various pharmacological activities such as antimicrobial, antioxidant; antimutagenic; anticarcinogenic; anti-inflammatory and etc. (Hemaiswaryaa et al. 2008). Piper betle was reported as good anticandidal agent since it contains bioactive components such as 4-chromanol (Kawsud et al. 2014), allylpyrocatechol (Dwivedi and Tripathi 2014), hydroxychavicol (Dwivedi and Tripathi 2014) and others.

$\mathrm{HC}$ has been reported to have antifungal activity. Although it is a useful natural compound for treating fungal infections, its high MIC prevents its effective use in clinical study. Therefore, it will be more effective to use $\mathrm{HC}$ in combination with conventional antifungal agents rather than used alone. To our knowledge, the in vitro phenomenon of synergism of HC with AMB and 5-FC against Candida spp. is reported here for the first time.

There are many models for experimental designs to measure such combination effects. One of the best known and very simple forms of such tests is the 'chequerboard' experiment in which a two dimensional array of serial concentrations of test compounds is used as the basis for calculation of a fractional inhibitory concentration index (FICI) (William et al. 2003). Applying these ideas to in vivo and clinical investigations of combination antifungal therapy is difficult, and no standards for interpretation of these data have been recommended. Analysis and comparison of results across in vivo and clinical studies requires careful consideration of the nature of pathogen, host, host immune status, study design, and study endpoints (Melissa et al. 2004). The effects observed in these models will not precisely apply to all aspects of the clinical. However, in vitro testing might suggest an effective antifungal at which the dose needed to achieve the desired effect. They also represent the best-controlled data we have and, upon review, they help us gain a better understanding of how these drugs might behave when used together. They also represent the best-controlled data we have and, upon review, they help us gain a better understanding of how these drugs might behave when used together. Thus, the only way to resolve some of these issues is to use the available in vitro and in vivo data to drive the design of carefully selected clinical studies of combination therapy in patients.

Combination between $\mathrm{HC}$ and AMB at 1:1, 1:2 and 2:1 ratio has resulted synergistic against most of species tested. While combination of $\mathrm{HC}$ with $5-\mathrm{FC}$ at $1: 1$, $1: 2$ and 2:1 ratio has resulted indifferent and antagonistic interaction. Combinationat 1:1 ratio between $\mathrm{HC}$ with AMB considered as a good antifungal combination since it showed synergistic effect at lowest ratio used in this study against Candida spp.

In the search for the mechanism of synergism between $\mathrm{HC}$ with $\mathrm{AMB}$, their individual antifungal modes of action need to be considered. From previous study, they reported that, $\mathrm{HC}$ alters the cell membrane structure, resulting in the disruption of the permeability barrier of microbial membrane structure (Nalina and Rahim 2007). While, polyenes antifungal are able to bind to ergosterol which is the main sterol in the fungal cell membrane. Enzymes in the ergosterol pathways are the targets of many antifungal agents. AMB binds with ergosterol, a component of fungal cell membranes, forming pores that cause rapid leakage of monovalent ions and subsequent fungal cell death (Mesa-Arango et al. 2012).

More importantly, our results showed that addition of $\mathrm{HC}$ to AMB could possibly enable reduced dosages of both agents and thus potentially reduced drug-associated toxicities which are frequently observed at the high dosages of using AMB alone (Van't Hof et al. 2000). Although the exact mechanism of interaction between $\mathrm{HC}$ and $\mathrm{AMB}$ is not known, it is possible that the simultaneous inhibition of different fungal cell targets occur. Combination of $\mathrm{HC} / \mathrm{AMB}$ appears to involve the disruption of cell membrane structure that cause rapid leakage of monovalent ions allows the passage of one or both agents. Thus, the synergism effect was observed in this combination.

5-FC resistant strains of Candida being clinically significant, thus there is a need for the development of new therapeutic agents especially in combination studies (Bondaryk et al. 2013). The results of these study provide evidence of antagonism effect between the combination of $\mathrm{HC}$ with $5-\mathrm{FC}$, and suggested that these agents should not be co-administered. A detailed mechanism explanation why the combination of $\mathrm{HC}$ and 5-FC antagonistic is unknown. It also can be suggested that it might be related to the disruption and changes in fungal cell membrane function due to the effect of $\mathrm{HC}$ at first place. According to Patel (1998), 5-FC is used in combination with other antifungal, such as AMB rather than used as monotherapy due to the increasing of drug resistance in combating fungal pathogens. In vitro data regarding the combination of both drugs against Candida species are numerous 
and somewhat contradictory showing antagonistic (Vandeputte et al. 2012).

\section{Conclusion}

In conclusion, $\mathrm{HC}$ has antifungal activity but a very high MIC value against oral Candida spp. However, the combination of $\mathrm{HC}$ with $\mathrm{AMB}$ exhibited a synergistic activity against all tested Candida species. Whereas, in combination with 5-FC exhibited indifference and antagonism activity. Therefore, the combination of HC/AMB effective at treating fungal infections and might be promising for future research into the pharmacological aspect. However, in vivo testing needs to be performed to support these findings.

\section{Authors' contributions}

CONZ and KNF make substantial contributions to conception and design, acquisition, analysis and interpretation of data. Dr WHH-A participated in drafting the article and analysis the data. All authors read and approved the final manuscript.

\section{Author details}

${ }^{1}$ Department of Oral Biology and Craniofacial Sciences, Faculty of Dentistry, University of Malaya, 50603 Kuala Lumpur, Malaysia. ${ }^{2}$ Department of Oral Biology and Biomedical Sciences, Faculty of Dentistry, University of Malaya, 50603 Kuala Lumpur, Malaysia.

\section{Acknowledgements}

This research is supported by Fundamental Research Grant Scheme (FP0342015A) and High Impact Research MoE Grant UM.C/625/1/HIR/MoE/DENT/17 from the Ministry of Education Malaysia.

\section{Competing interests}

The authors declare that they have no competing interests.

Received: 14 July 2016 Accepted: 27 September 2016

Published online: 03 October 2016

\section{References}

Akins RA, Sobel JD (2009) Antifungal targets, mechanisms of action, and resistance in Candida albicans. In: Mayers DL (ed) Antimicrobial drug resistance. Humana Press, New York, pp 347-407

Bokor-Bratic MB (2008) Oral candidiasis-adhesion of non-albicans Candida species. Proc Nat Sci 114:69-78

Bondaryk M, Kurzątkowski W, Staniszewska M (2013) Antifungal agents commonly used in the superficial and mucosal candidiasis treatment: mode of action and resistance development. Postepy Dermatol Alergol 30(5):293-301
Clinical and Laboratory Standards Institute (CLSI) (2008) Reference method for broth dilution antifungal susceptibility testing of filamentous fungi; approved standard-second edition. CLSI document M38-A2. Wayne, PA

Cuenca-Estrella M (2004) Combinations of antifungal agents in therapy-what value are they? J Antimicrob Chemother 54(5):854-869

Dwivedi V, Tripathi S (2014) Review study on potential activity of Piper Betle. J Pharmacogn Phytochem 3(4):93-98

Fridkin SK, Jarvis WR (1996) Epidemiology of nosocomial fungal infections. Clin Microbial Rev 9(4):449-511

Ghannoum MA, Rice LB (1999) Antifungal agents: mode of action, mechanism of resistance, and correlation of these mechanisms with bacterial resistance. Clin Microbiol 12(4):501-507

Harun WHA, Razak WFA, Musa MY (2014) Growth inhibitory response and ultrastructural modification of oral-associated candidal reference strains (ATCC) by Piper betle L. extract. Int J Oral Sci 6(1):15-21

Hemaiswaryaa S, Kruthiyentib AK, Doblea M (2008) Synergism between natural products and antibiotics against infectious diseases. Phytomedicine 15(8):639-652

Johnson MD, MacDougall C, Ostrosky-Zeichner L, Perfect JR, Rex JH (2004) Combination antifungal therapy. Antimicrob Agents Chemother 48(3):693-715

Kawsud P, Puripattanayong J, Teanpaisan R (2014) Screening for anticandidal and antibiofilm activity of some herbs in thailand. Trop J Pharm 13(9):1495-1501

Melissa DJ, Conan MD, Luis OZ, John RP, John HR (2004) Combination antifungal therapy. Antimicrob Agents Chemother 48(3):693-715

Mesa-Arango AC, Scorzoni L, Zaragoza O (2012) It only takes one to do many jobs: amphotericin B as antifungal and immunomodulatory drug. Front Microbiol 286(3):1-10

Nalina T, Rahim ZHA (2007) The crude aqueous extract of Piper betle L. and its antibacterial effect towards Streptococcus mutans. Am J Biochem Biotechnol 3(1):10-15

Patel R (1998) Antifungal agents. Part I. Amphotericin B preparations and flucytosine. Mayo Clin Proc 73(12):1205-1225

Sharma S, Khan IA, Ali I, Ali F, Kumar M, Kumar A, Hohri RK, Abdullah ST, Bani S, Pandey A, Suri KA, Gupta BD, Satti NK, Dutt P, Qazi GN (2009) Evaluation of the antimicrobial, antioxidant and anti-inflammatory activities of hydroxychavicol for its potential use as an oral care agent. Antimicrob Agents Chemother 53(1):216-222

Sugar AM (1986) The polyene macrolide antifungal drugs. In: Peterson PK, Verhoef J (eds) Antimicrob agents chemother. Elsevier, Amsterdam, pp 229-244

Van't Hof W, Reijnders IM, Helmerhorst EJ, Walgreen-Weterings E, SimoonsSmit IM, Veerman EC, Amerongen AVN (2000) Synergistic effects of low doses of histatin 5 and its analogues on amphotericin B anti-mycotic activity. Antonie Van Leeuwenhoek 78(2):163-169

Vandeputte P, Ferrari S, Coste AT (2012) Antifungal resistance and new strategies to control fungal infections. Int J Microbiol 2012:713687

Vermes A, Guchelaar HJ, Dankert J (2000) Flucytosine: a review of its pharmacology, clinical indications, pharmacokinetics, toxicity and drug interactions. J Antimicrob Chemother 46(2):171-179

William JS, David AS, David WD (2003) Combination and sequential antifungal therapy for invasive aspergillosis: review of published in vitro and in vivo interactions and 6281 clinical cases from 1966 to 2001. Clin Infect Dis 37(Suppl 3):S188-S224 